\title{
Use of Artificial Intelligence to Improve Diabetes Outcomes in Patients Using Multiple Daily Injections Therapy
}

\author{
Gregory P. Forlenza, MD
}

Keywords: Type 1 diabetes, Artificial intelligence, Multiple daily injections, Automated decision support.

\section{Introduction}

T HE STATE OF TYPE 1 DIABETES (T1D) therapy in 2019 is a complex mixture of groundbreaking technological innovations and incompletely realized improvements to clinical care. In the past several years, we have seen the commercial approval of the first hybrid closed loop (HCL) artificial pancreas (AP) system, ${ }^{1-3}$ the first real-time factorycalibrated continuous glucose monitor (CGM), ${ }^{4,5}$ and predictive low glucose suspend systems, ${ }^{6,7}$ as well as approvals for the first interoperable continuous subcutaneous insulin infusion (CSII) pump and CGM configurations. Previous registry research has demonstrated that insulin pump users experience significantly lower hemoglobin A1c (HbA1c) values than multiple daily injection (MDI) users and that patients assessing their glycemic status with CGM experience lower HbA1c values than those using self-monitoring of blood glucose alone. ${ }^{8,9}$ Data from HCL AP technology have consistently shown significant glycemic improvements in both short-term and long-term studies. ${ }^{10-13}$ Accordingly, we should expect that the proliferation of these technologies would bring a notable improvement in glycemic control across all patient populations.

The recent T1D exchange clinical registry analysis by Foster thus comes as a stark reminder that much work still needs to be done to improve clinical outcomes across the broader patient population. ${ }^{14}$ This report on $>22,000$ persons with T1D builds on the previous article by Miller et al. ${ }^{15}$ and documents that between the 2010-2012 and 2016-2018 periods, CSII pump use increased from $57 \%$ to $63 \%$ and CGM use increased from $7 \%$ to $30 \%$ with an exponential increase in 2013-2014. Despite this, the adjusted mean $\mathrm{HbA} 1 \mathrm{c}$ increased from $7.8 \%$ in $2010-2012$ to $8.4 \%$ in 2016-2018, with the largest increase seen among adolescents and young adults. Sub-analysis by device use best highlighted in a separate commentary by Rodbard ${ }^{16}$ shows that CGM users have HbA1c values $0.4 \%$ to $1.0 \%$ lower than nonusers and pump users have $\mathrm{HbA} 1 \mathrm{c}$ values $0.2 \%$ to $0.8 \%$ lower than MDI users. Foster notes, in particular, that among CGM users, HbA1c levels were similar whether the person was using CSII or MDI for insulin delivery while Rodbard notes that differences in control between different racial/ethnic and income groups may be mediated by access to technology.

In their discussions, both Foster and Rodbard were unable to conclude why glycemic control had worsened across the registry despite increased use of technology, although Rodbard speculated that it may be due to changes in the population regarding study sites, racial/ethnic groups, and income groups. The Foster analysis attempted to control for duration of diabetes and failed to identify changes as due to differences over time in racial/ethnic groups, income status, or other known confounding factors. Rodbard, however, notes that some of these effects may be nonlinear and thus not readily identified using multiple linear regression. In searching for other possible confounders, it is notable that BMI z-score was unchanged between time periods. Comparing the Miller 2010-2012 data with the Foster 20162018 data, within CGM users all age groups had worse $\mathrm{HbA} 1 \mathrm{c}$ in the latter period, with an even larger effect among CGM nonusers. Among pump users, HbA1c was roughly unchanged across age groups, whereas among MDI users, $\mathrm{HbA} 1 \mathrm{c}$ increased mostly in the adolescents and younger adults.

One potentially important finding from the Foster article that may be easily missed is that most participants never downloaded their device data (meters, CGMs, or CSII pumps) at home, and other than using the Dexcom Share feature, use of mobile applications was very uncommon. ${ }^{14}$ Over $71 \%$ of participants not using CGM reported never downloading their blood glucose meter outside of the doctor's office, $51 \%$ of CGM users reported never downloading

University of Colorado Denver, Barbara Davis Center, Pediatric Endocrinology, Aurora, Colorado. 
their CGM outside of the doctor's office, $60 \%$ reported never downloading their CSII pump outside of the doctor's office, and $84 \%$ reported never using a mobile medical application to assist with diabetes. The reasons for failure to download devices were varied and included lack of awareness of downloading, time and difficulty with downloading, difficulty understanding the data, and not finding the data useful.

Taken together, these findings on glycemic control trends and patient use of digital diabetes data may help us understand why increased technology use has not corresponded with improved glycemic control. Although patients have greater amounts of diabetes data, they are not fully utilizing this information to optimize diabetes therapy.

This brings us to the frontier of automated decision support (ADS) systems, or the use of computer-based artificial intelligence technology to provide feedback or guidance to patients or clinicians. In the case of CSII users, ADS could provide recommendations to adjust pump settings, whereas for MDI users, it could suggest changes to basal insulin, carbohydrate ratios, or even sliding scale dosing. In this commentary, we review the existing literature on this emerging area of therapy, focusing on applications to patients receiving MDI therapy.

\section{Rationale for ADS Technology}

The fundamental idea behind ADS for patients using MDI therapy is to utilize their digital diabetes data, likely from CGM and Bluetooth-enabled smart pens, along with a computerbased dosing controller, likely on a smartphone, to optimize insulin dosing on a daily or weekly basis (Fig. 1). Such a system would provide the benefit of digital data review and dosing optimization without the burden of patients or caregivers needing to visualize their own data and adjust their own insulin dosing. It can be viewed as a form of closed-loop control for MDI patients where the control is optimized less frequently than the every 5-minute-basis used in AP technology.
Some may be concerned that an ADS system running without physician oversight may provide unsafe recommendations to patients. The foundation for this concern has been addressed in a novel study by Nimri et al. ${ }^{17}$ This study evaluated the insulin dosing adjustments made by 26 physicians from 16 centers across Europe, Israel, and South America for pump settings based on CGM data. These adjustments were compared against the DreaMed Advisor Pro (DreaMed Diabetes Ltd., Petah Tikva, Israel). The study found that there was only $41 \%-45 \%$ agreement among physicians in the direction of adjustment to basal rates, carbohydrate ratios, and correction factors across the 15 cases studied. The ADS algorithm provided similar directional agreement to that of the clinicians, with the magnitude of dosing change equal to or less than that recommended by the clinicians. Many conclusions can be drawn from this study, although a common conclusion is that insulin dosing adjustment is both an art and a science, and various expert clinicians may provide a range of different recommendations to a given situation. An ADS tool that provides recommendations similar to experts may simply be viewed as one of a variety of expert opinions to a given glycemic case.

The IBM Watson Research Center (Yorktown Heights, $\mathrm{NY}$ ) has begun qualitatively investigating what would be needed in a patient-centered ADS system. ${ }^{18}$ They report results from a year-long study of 26 people with T1D and their decision processes. The authors identified several difficulties encountered by patients in making their own diabetes decisions including identifying causal factors, identifying intensity of causal factors, identifying solutions, staying accountable to priorities, identifying current trends, estimating individualized outcomes, too much information, and inconvenient access to information. As solutions to these challenges, the authors concluded that ADS systems should automate as much data entry as possible (e.g., CGM data), but should allow for patient-centered data entry as well (e.g., exercise, stress). They also found that the analysis should be

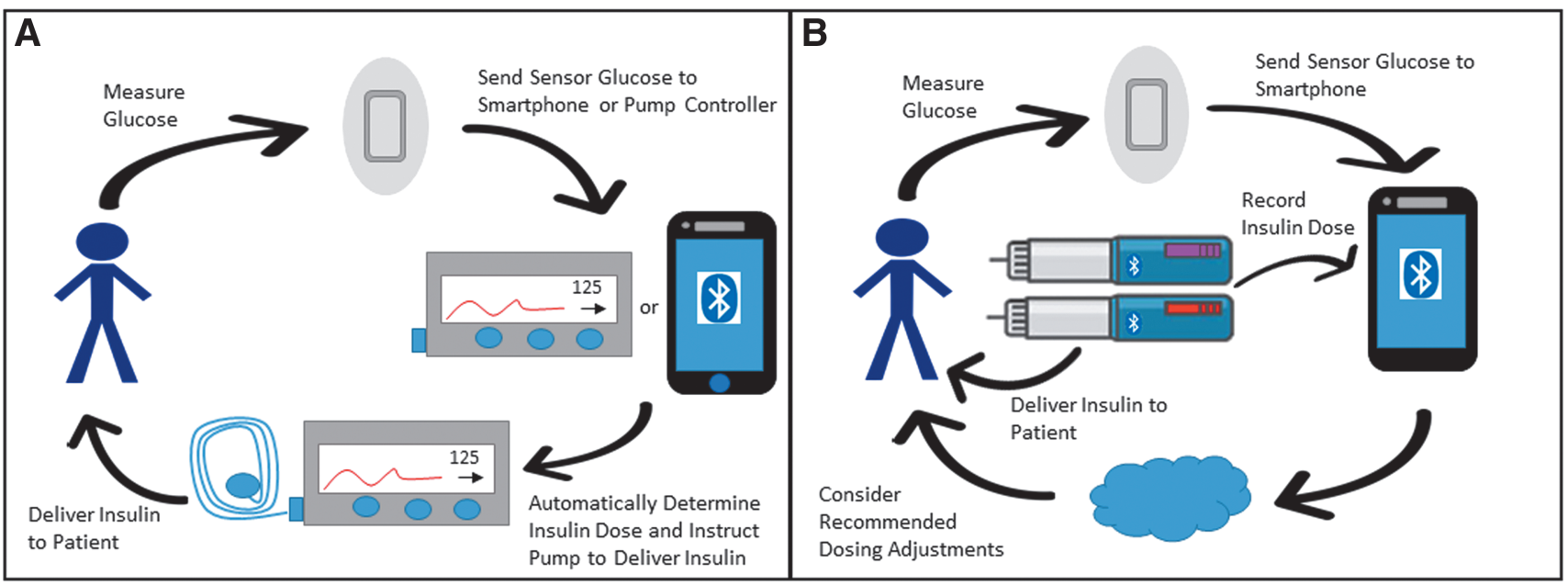

FIG. 1. Automated insulin dosing adjustment with HCL AP and MDI ADS designs. (A) depicts a HCL AP system whereby the CGM relays glycemic values to a smartphone or pump-based dosing controller, the controller then regulates pump basal rates, and the pump delivers the insulin to the patient. (B) depicts MDI ADS whereby the CGM sends glycemic values to a smartphone, smartpen(s) communicate insulin dosing to a smartphone, and a smartphone-based advisor conveys dosing recommendations to a patient and/or provider. ADS, automated decision support; AP, artificial pancreas; CGM, continuous glucose monitor; HCL, hybrid closed loop; MDI, multiple daily injection. 
individualized with the output appearing as natural language to be better understood by patients.

\section{Studies on ADS Technology}

Artificial intelligence and ADS are both very broad topics with many potential applications to the health of patients with T1D as well as patients with type 2 diabetes (T2D). Work is being done on physician-focused ADS systems to help providers identify whom to screen for T2D, how to optimize complication reduction and guideline compliance with routine studies for patients with T1D and T2D, and automated reading of images screening for diabetic retinopathy. ${ }^{19-22}$ Although these frontiers are exciting, they are more focused on provider efficiency rather than patient-centered application of ADS technologies. To date, there are limited clinical trial data on the use of ADS for MDI patients. Clinicaltrials.gov lists only one ongoing study of ADS for MDI patients, a trial of the DreaMed MD-Logic Switch Advisor to provide recommendations for switching patients between MDI and CSII therapy. ${ }^{23}$ The reasons for this limited level of clinical research are unclear; however, they could be related to the current focus on AP development, poor use of apps by patients in the real world setting, or the lack of suitable Bluetooth-enabled insulin pens to fully support insulin inputs into an MDI ADS system.

Breton conducted a prospective study of 24 subjects with T1D using either CSII or MDI therapy with carbohydrate counting. ${ }^{24}$ Subjects completed two randomized crossover 48-h visits with either usual care or ADS with the University of Virginia Decision Support System (UVA DSS). The UVA DSS provided automated insulin titration, bolus calculation, and carbohydrate treatment advice. This study found that use of the DSS significantly reduced glycemic variability and hypoglycemia exposure without increasing average CGM values. The detailed analysis showed that both MDI and CSII subjects experienced improved glycemic control with use of the UVA DSS. An important caveat, however, for this finding is that the MDI subjects were required to use the MySugr app (MySugr, Vienna, Austria) to enter all carbohydrate and bolus information. As the T1D exchange data have shown, real-world use of such apps is extremely rare outside of the research setting. ${ }^{14}$ Thus although these preliminary data are promising, adoption of this technology in clinical care would require a substantial behavioral change in the MDI patient population.

Reddy has published results on a 10-day prospective nonrandomized single-arm pilot study of the Advanced Bolus Calculator for Diabetes (ABC4D) for patients using MDI therapy. ${ }^{25}$ The ABC4D system uses case-based reasoning to provide real-time insulin advice through a smartphone application. This study showed that $>6$ weeks of use, the number of postprandial hypoglycemic episodes decreased significantly. The percentage time in the target range of 70$180 \mathrm{mg} / \mathrm{dL}$ increased from $55 \%$ to $61 \%$, although the study was not powered to detect a difference of this size. The mean sensor glucose also appeared to decrease from 171 to $164 \mathrm{mg} / \mathrm{dL}$, although again the study was not powered to detect a decrease of this size.

Several studies have evaluated the use of ADS for CSII patients. Although HCL systems may make such approaches unnecessary in the near future, these studies still point to the impact of ADS to improve care of open loop systems. Earlier we discussed the Nimri study on the DreaMed advisor and its directional agreement with a diverse group of clinicians. ${ }^{17}$ Herrero conducted an in silico validation of a basal insulin ADS tool for patients on CSII therapy. ${ }^{26}$ This virtual study showed that use of the ADS tool could significantly improve time in target range and reduce hypoglycemia over a 4-month period. Cappon developed a neural network to optimize and personalize meal bolus calculations using CGM data. ${ }^{27}$ This system was tested in an in silico trial of 100 meals. Its use was found to significantly decrease the blood glucose risk index after meals. Dassau investigated the use of ADS to optimize the parameters used to control a HCL AP system. ${ }^{28}$ This study looked at 37 adults with T1D undergoing a randomized crossover study of HCL therapy with either conventional pump settings or pump settings optimized by ADS. It showed no change in time in target range during a brief observational period of $27 \mathrm{~h}$ without any change in hypoglycemia.

Synthesis of these small trial results indicates that ADS has the potential to improve basal as well as prandial insulin control for people on MDI as well as CSII therapy. The benefits seen from these approaches appear somewhat similar to the benefits seen for AP therapy: automation can decrease glycemic variability, reduce hypoglycemia, and potentially improve time in target range. These benefits, however, would still be limited by the need to wear CGM, the need to carbohydrate count, and the need to routinely use and interact with the ADS application providing the information. Such limitations are similar to those seen for HCL therapy, although without the need to wear and maintain a CSII pump. Thus, education, physician support, and behavioral modification will be essential to realize the full benefits from this technology in a real-world setting.

\section{Guidelines on ADS Technology}

As ADS is a very new and emerging technology, there is relatively little guidance for its clinical use available for clinicians, patients, and diabetes educators. The American Diabetes Association (ADA) has in 2019 released the first set of diabetes technology standards as a separate chapter. ${ }^{29}$ The ADA guidelines discuss insulin pens, CSII pumps, blood glucose meters, CGMs, and HCL AP systems; however, diabetes apps and ADS technology are not addressed. In 2018, the International Society for Pediatric and Adolescent Diabetes (ISPAD) released its first set of diabetes technology guidelines as a separate chapter. ${ }^{30}$ These guidelines contain a section specifically addressing diabetes apps, ADS, and bolus calculators. The executive summary of the ADS from the guidelines was "Automated algorithmic adjustment of openloop pump settings and insulin dosing parameters is an emerging area of research and clinical care in diabetes technology (E). The first system for automated dosing adjustment with health-care provider approval has just received regulatory approval." 30 The ISPAD guidelines also review the state of ADS research at the time of writing and discuss future applications to MDI, CSII, and even HCL therapy.

\section{Commercially Available Systems}

Identifying what systems for ADS are commercially available is difficult and any list may be incomplete. The DreaMed Advisor Pro was approved by the FDA on June 12, 
2018. ${ }^{31}$ This system is approved as a decision-support software intended for assisting health care professionals in the management of CSII patients using CGM and not on HCL systems. The diabetes management platform One Drop announced the release of an ADS tool on September 28, 2018. This support tool uses a machine-learning algorithm to predict $B G$ values for patients with noninsulin-treated T2D. In silico testing of this model has shown that $91 \%$ of predictions fell within $50 \mathrm{mg} / \mathrm{dL}$ at various time thresholds and that the mean absolute error of predictions was $21.3 \mathrm{mg} / \mathrm{dL}^{32}$ One Drop's ADS platform has also been tested using both inhaled and injected insulin with preliminary results showing that use of ADS may be associated with HbA1c improvement among users of inhaled insulin. ${ }^{33}$ Complete presentation of these study results as well as further work on patients with T1D is still pending.

\section{Future Developments}

As already discussed, earlier work on ADS systems for patients on MDI therapy shows that algorithms can impart benefits including reduction of hypoglycemia, reduction in glycemic variability, and presumably improved overall glycemic control. Patients will need to provide CGM glycemic data, insulin dosing data, and likely carbohydrate ingestion data to fully benefit from this avenue of technology. The latest T1D exchange data demonstrate exponential growth in CGM use in all age groups, including among MDI users. As CGM systems move toward factory calibration, longer duration of wear, and smaller size, use of this technology will continue to expand. The other piece of technology necessary for full adoption of ADS in MDI users is Bluetooth-enabled insulin pens. The Companion Medical InPen is a Bluetooth-enabled pen for aspart and lispro rapid acting insulins. Both Lilly and Novo Nordisk are reported to be developing Bluetoothenabled smart pens that could also be used in ADS systems.

\section{Conclusions}

Expansion of technology to aid patients in care of T1D has not yet fully realized the goal of improved glycemic control. Application of AI principles to patients on MDI therapy in the form of ADS has shown early promise to help bridge this gap and improve control in this population. Many different systems and platforms are under development, and as additional tools become available, it will be incumbent on providers to educate and train their patients in the use of this technology. Additional burden reduction through improved CGM technology and Bluetooth-enabled smart pens may further facilitate expansion of this technology. Growth in this area of care will be vital over the next few years to ensure that all patients with diabetes fully realize the benefits of automation and artificial intelligence.

\section{Acknowledgment}

Effort for this project was supported by a National Institutes of Health K12 grant (K12DK094712).

\section{Author Disclosure Statement}

G.P.F. conducts research supported by Medtronic, Dexcom, Abbott, Tandem and Insulet and is a speaker/consultant for Medtronic, Dexcom, and Tandem.

\section{References}

1. Garg SK, Weinzimer SA, Tamborlane WV, et al.: Glucose outcomes with the in-home use of a hybrid closed-loop insulin delivery system in adolescents and adults with type 1 diabetes. Diabetes Technol Ther 2017;19:155-163.

2. Bergenstal RM, Garg S, Weinzimer SA, et al.: Safety of a hybrid closed-loop insulin delivery system in patients with type 1 diabetes. JAMA 2016;316:1407-1408.

3. Forlenza GP, Pinhas-Hamiel O, Liljenquist DR, et al.: Safety evaluation of the MiniMed $670 \mathrm{G}$ system in children 7-13 years of age with type 1 diabetes. Diabetes Technol Ther 2019; 21:11-19.

4. Wadwa RP, Laffel LM, Shah VN, Garg SK: Accuracy of a factory-calibrated, real-time continuous glucose monitoring system during 10 days of use in youth and adults with diabetes. Diabetes Technol Ther 2018;20:395-402.

5. Shah VN, Laffel LM, Wadwa RP, Garg SK: Performance of a factory-calibrated real-time continuous glucose monitoring system utilizing an automated sensor applicator. Diabetes Technol Ther 2018;20:428-433.

6. Forlenza GP, Li Z, Buckingham BA, et al.: Predictive lowglucose suspend reduces hypoglycemia in adults, adolescents, and children with type 1 diabetes in an at-home randomized crossover study: results of the PROLOG trial. Diabetes Care 2018;41:2155-2161.

7. Wood MA, Shulman DI, Forlenza GP, et al.: In-clinic evaluation of the MiniMed 670G system "suspend before low" feature in children with type 1 diabetes. Diabetes Technol Ther 2018;20:731-737.

8. Sherr JL, Hermann JM, Campbell F, et al.: Use of insulin pump therapy in children and adolescents with type 1 diabetes and its impact on metabolic control: comparison of results from three large, transatlantic paediatric registries. Diabetologia 2016;59:87-91.

9. DeSalvo DJ, Miller KM, Hermann JM, et al.: Continuous glucose monitoring (CGM) and glycemic control among youth with type 1 diabetes (T1D): international comparison from the T1D exchange and DPV initiative. Pediatr Diabetes 2018;19:1271-1275.

10. Stone MP, Agrawal P, Chen X, et al.: Retrospective analysis of 3-month real-world glucose data after the MiniMed 670G system commercial launch. Diabetes Technol Ther 2018;20:689-692.

11. Weisman A, Bai JW, Cardinez M, et al.: Effect of artificial pancreas systems on glycaemic control in patients with type 1 diabetes: a systematic review and meta-analysis of outpatient randomised controlled trials. Lancet Diabetes Endocrinol 2017;5:501-512.

12. Bekiari E, Kitsios K, Thabit H, et al.: Artificial pancreas treatment for outpatients with type 1 diabetes: systematic review and meta-analysis. BMJ 2018;361:k1310.

13. Dai X, Luo ZC, Zhai L, et al.: Artificial pancreas as an effective and safe alternative in patients with type 1 diabetes mellitus: a systematic review and meta-analysis. Diabetes Ther 2018;9:1269-1277.

14. Foster NC, Beck RW, Miller KM, et al.: State of type 1 diabetes management and outcomes from the T1D exchange in 2016-2018. Diabetes Technol Ther 2019;21:66-72.

15. Miller KM, Foster NC, Beck RW, et al.: Current state of type 1 diabetes treatment in the U.S.: updated data from the T1D exchange clinic registry. Diabetes Care 2015;38:971-978.

16. Rodbard D: State of type 1 diabetes care in the United States in 2016-2018 from T1D exchange registry data. Diabetes Technol Ther 2019;21:62-65. 
17. Nimri R, Dassau E, Segall T, et al.: Adjusting insulin doses in patients with type 1 diabetes who use insulin pump and continuous glucose monitoring: variations among countries and physicians. Diabetes Obes Metab 2018;20:2458-2466.

18. Sun S, Costello KL: Designing decision-support technologies for patient-generated data in type 1 diabetes. AMIA Annu Symp Proc 2017;2017:1645-1654.

19. Rigla M, Garcia-Saez G, Pons B, Hernando ME: Artificial intelligence methodologies and their application to diabetes. J Diabetes Sci Technol 2018;12:303-310.

20. Perez-Gandia C, Garcia-Saez G, Subias D, et al.: Decision support in diabetes care: the challenge of supporting patients in their daily living using a mobile glucose predictor. J Diabetes Sci Technol 2018;12:243-250.

21. Dankwa-Mullan I, Rivo M, Sepulveda M, et al.: Transforming diabetes care through artificial intelligence: the future is here. Popul Health Manag 2018 [Epub ahead of print]; doi: 10.1089/pop.2018.0129.

22. Contreras I, Vehi J: Artificial intelligence for diabetes management and decision support: literature review. J Med Internet Res 2018;20:e10775.

23. Personalized automated determination of insulin pump setting for subjects with type 1 diabetes switching from MDI to pump therapy and vice versa- safety and efficacy feasibility study. https://www.clinicaltrials.gov/ct2/show/ NCT03574558?term=automated+decision+support\&cond= diabetes\&rank=5 (accessed February 27, 2019).

24. Breton MD, Patek SD, Lv D, et al.: Continuous glucose monitoring and insulin informed advisory system with automated titration and dosing of insulin reduces glucose variability in type 1 diabetes mellitus. Diabetes Technol Ther 2018;20:531-540.

25. Reddy M, Pesl P, Xenou M, et al.: Clinical safety and feasibility of the advanced bolus calculator for type 1 diabetes based on case-based reasoning: a 6-week nonrandomized single-arm pilot study. Diabetes Technol Ther 2016;18:487-493.
26. Herrero P, Bondia J, Gimenez M, et al.: Automatic adaptation of basal insulin using sensor-augmented pump therapy. J Diabetes Sci Technol 2018;12:282-294.

27. Cappon G, Vettoretti M, Marturano F, et al.: A neuralnetwork-based approach to personalize insulin bolus calculation using continuous glucose monitoring. J Diabetes Sci Technol 2018;12:265-272.

28. Dassau E, Brown SA, Basu A, et al.: Adjustment of openloop settings to improve closed-loop results in type 1 diabetes: a multicenter randomized trial. J Clin Endocrinol Metab 2015;100:3878-3886.

29. American Diabetes Association: 7. Diabetes technology: standards of medical care in diabetes-2019. Diabetes Care 2019;42:S71-S80.

30. Sherr JL, Tauschman M, Battelino T, et al.: ISPAD clinical practice consensus guidelines 2018 diabetes technologies. Pediatr Diabetes 2018;19:302-325.

31. DreaMed Advisor Pro: FDA; 2018 [updated June 12, 2018]. Available from: https://www.accessdata.fda.gov/cdrh_docs/ pdf17/DEN170043.pdf.

32. Goldner DR, Osborn CY, Sears LE, et al.: A machinelearning model accurately predicts projected blood glucose. Diabetes 2018;67:46-LB.

33. Hirsch A, Heyman M, Raymond J, et al.: A pragmatic randomized control trial evaluates one drop with inhalable vs. injectable insulin (abstract). Diabetes Technol Ther 2019;21(S1):327.

Address correspondence to:

Gregory P. Forlenza, MD

Barbara Davis Center

University of Colorado Denver

1775 Aurora CT

MS A140

Aurora, CO 80045

E-mail: gregory.forlenza@ucdenver.edu 